\title{
Technological innovation in the sentinel node procedure: towards 3-D intraoperative imaging
}

\author{
Renato A. Valdés Olmos • Sergi Vidal-Sicart • \\ Omgo E. Nieweg
}

Published online: 29 April 2010

(C) Springer-Verlag 2010

The concept of the sentinel node procedure is based on the existence of an orderly and predictable pattern of lymphatic drainage to a regional lymph node basin, and on the functioning of lymph nodes on a direct drainage pathway as effective filters for tumour cells. When Dr. Donald Morton described the modern concept of lymphatic mapping in melanoma in 1992, he also introduced the technique of intraoperative visualization of the sentinel node using blue dye [1]. With the addition of radioactive colloids and gamma probe detection in 1993 the acoustic signals, corresponding with the detection of radioactivity, were incorporated into the sentinel node search in the operating room [2]. In the last two decades, this combination of visual and auditory signals has been a fundament of the intraoperative identification of sentinel nodes in patients with melanoma or breast cancer. The combination of "hot" and "blue" in the operating room has been successful to localize the sentinel node in the expected lymph drainage basin. The blue dye is less effective in areas of aberrant drainage that may be indicated preoperatively by lymphoscintigraphy. Blue dye has also a limited value in deep nodal basins.

Against this background, a new device was developed based on the gamma ray detection probe. This device combines acoustic signals with 3-D imaging for the localization of the sentinel node in the operating room. This innovative tool may solve various limitations of the current generation of probes [3].

R. A. Valdés Olmos $(\bowtie) \cdot$ O. E. Nieweg

Netherlands Cancer Institute, Antoni van Leeuwenhoek Hospital, Amsterdam, The Netherlands

e-mail: r.valdes@nki.nl

S. Vidal-Sicart

Hospital Clínic Barcelona, University of Barcelona,

Barcelona, Spain
Eyes and ears in the sentinel node procedure

In this millennium, the sentinel node concept has expanded to more complex applications with sophisticated demands concerning tracer administration and imaging. Since its introduction in 1992 for melanoma, lymphatic mapping with a simple intradermal injection and planar images enabled the identification of the sentinel node in virtually all cases because of the swift and superficial lymphatic drainage. In spite of a large variability of the injection techniques, tracer administration in patients with palpable breast cancer is relatively easy and a sentinel node in the axilla is harvested in the majority of patients. For nonpalpable breast tumours, new demands for injection were raised, especially when the sentinel node procedure was combined with radioguided excision of the primary tumour. Cooperation with radiologists was established to enable tracer injection into the tumour, guided by ultrasound or stereotaxis. The injection in or near the tumour is accompanied by a higher incidence of sentinel nodes outside the axilla and their removal demands more time and expertise of surgeons. Only $80 \%$ of the sentinel nodes of the internal mammary chain can be found in the operating room [4]. The hard to reach location of some of these sentinel lymph nodes and their frequent lack of blue staining may explain why the surgical identification rate in this basin is lower than in the axilla.

The same evolution has been observed for other areas of application of the sentinel node procedure. European countries have the largest experience with the sentinel node procedure in urology. The procedure has been used in penile cancer, prostate cancer, bladder cancer, testicular carcinoma and renal cell carcinoma. Penile cancer was the first application and both injection technique and image acquisition were validated in a manner similar to the 
approach in melanoma. The combination of the probe and the blue dye was also successful in patients with this cancer type. In patients with prostate cancer, the radioactive tracer injection needs to be guided by transrectal ultrasound and imaging demands SPECT/CT for accurate sentinel node localization in the pelvis and retroperitoneum. Intraoperative use of blue dye is not very effective and sentinel nodes are typically localized using only acoustic signals. This approach may lead to a prolongation of the operation, particularly when laparoscopy is employed.

In gynaecology, the first validated application concerned vulvar cancer. Lymph from this malignancy superficially drains to the groins. The combination of lymphoscintigraphy, a gamma probe and blue dye usually allows the identification of the sentinel node. Blue dye is less successful for deeper situated malignancies such as cancer of the cervix and the endometrium, and the gynaecologist depends on the gamma probe, similar to the situation in prostate cancer.

Finally, in gastrointestinal malignancies the sentinel node procedure has been characterized by more sophisticated approaches. Lymphoscintigraphy to preoperatively localize sentinel nodes and exclude aberrant drainages is very appealing, but the radioactive tracer injection demands the use of endoscopy. This is the case in gastric cancer, which has been extensively studied in Japan and other Asiatic countries. Colon cancer has been studied in a variety of ways, using in vivo and ex vivo techniques, but few investigators have used lymphoscintigraphy. Besides radioactive colloids, vital dyes and fluorescent tracers have also been employed for lymphatic mapping in this malignancy.

\section{Technological innovation}

The sentinel node procedure was started in the 1990s on the basis of two nuclear medicine devices: the gamma camera for preoperative lymphoscintigraphy and the gamma probe for intraoperative sentinel node detection. Radiolabelled colloids were already available for daily practice. The use of simultaneous transmission scanning using a ${ }^{57} \mathrm{Co}$ or ${ }^{99 \mathrm{~m}} \mathrm{Tc}$ flood source was incorporated subsequently to give nuclear physicians and surgeons more anatomical information about the location of the sentinel node.

The introduction of new technological possibilities for sentinel node detection has only properly occurred in the last few years [5]. With the new generation of large field gamma cameras, the functional information from SPECT can be combined with the morphological information from $\mathrm{CT}$ by applying both techniques in one session. The resulting SPECT/CT fused images depict sentinel nodes in an anatomical 2-D and 3-D landscape providing a helpful roadmap for surgeons. In recent years, SPECT/CT has been used in melanoma and breast cancer patients with unusual or complex drainage. This is the case in melanomas of the neck or the upper part of the trunk and in breast cancer in patients with drainage outside the axilla. SPECT/CT may also visualize sentinel nodes in the axilla if no lymph nodes are visualized on planar images. Furthermore SPECT/CT is becoming essential to localize sentinel nodes in locations such as the pelvis, retroperitoneum and upper abdomen in gastrointestinal, gynaecological and urological malignancies.

A new generation of portable gamma cameras was recently introduced for intraoperative use. These portable devices can be oriented to the surgical targets in the operating room based on the anatomical landmarks previously established by SPECT/CT fused images [6]. In both open surgery and laparoscopy, this small device may be used in combination with the standard gamma probe or the laparoscopic probe. The portable gamma camera makes a strong contribution by indicating the direction of the sentinel node on skin by means of laser or external radioactive pointers. This is valuable for instance when the melanoma is situated in the head or neck for sentinel nodes near the injection site, which is a difficult location for the gamma probe on its own. The portable gamma camera is also helpful to exclude a remaining hot spot after a sentinel node has been harvested. In the abdomen or pelvis, the portable gamma camera can orientate the surgeon by displaying on the monitor the area where the sentinel node is located, guided by a radioactive source fixed on the laparoscopic probe. However, in many cases with lymphatic drainage to the dorsal pelvic areas and retroperitoneal area it is very difficult to identify the sentinel node. To overcome this limitation, the information provided by a freehand SPECT device by combining the acoustic and visual signals offers a new approach that deserves to be evaluated.

\section{Towards intraoperative 3-D imaging}

In this issue, Wendler et al. introduce an ingenious device combining a spatial localization system and two tracking targets to be fixed on a gamma probe and on the patient [3]. The localization system consists of an optical camera and an infrared localization device. Following a protocol of movements with the probe, 3-D images can be generated with this freehand SPECT device and these are visualized on the screen. The images can be displayed in real time and information on the depth of a sentinel node is available. The feasibility of 3-D sentinel node localization using the freehand SPECT system was assessed in breast cancer patients scheduled for the sentinel node procedure. Togeth- 
er with the validation for sentinel node localization in breast cancer, the first evaluation of this new modality is also a proof of principle to demonstrate its feasibility as a possible tool for the procedure in a wider sense. There are various important aspects to underline in this initial experience. Firstly, the freehand 3-D appears to be a device that is easy to handle in the operating room. Secondly, the accuracy of the system depends on the sentinel node uptake; the more intense the uptake, the easier the 3-D imaging. Furthermore, the positioning device of the system can potentially be attached to the existing, commercially available gamma probes. This may encourage its introduction into the operating theatre. The freehand SPECT device can combine the traditional acoustic signals of the gamma probe with the new 3-D visualization. This feature together with the realtime depth information that the system may provide would potentially expand its application in the sentinel node procedure in oncology, particularly for malignancies with deep lymphatic drainage. For instance, the device would be of great value for laparoscopy-guided procedures in urological and gynaecological malignancies. A combination with a portable gamma camera in this field is possible. Finally, a new generation of wireless gamma probes has recently been introduced. The use of these probes with the freehand SPECT device may develop into a potent tool not only in sentinel node retrieval but also for radioguided resection of primary tumours and isolated metastases.
There are reasons to believe that the increasing technological innovation of nuclear medicine is opening new frontiers and dimensions in radioguided surgery. Better eyes and ears are always welcome.

\section{References}

1. Morton DL, Wen DR, Wong JH, Economou JS, Cagle LA, Storm FK, et al. Technical details of intraoperative lymphatic mapping for early stage melanoma. Arch Surg 1992;127:392-9.

2. Krag DN, Weaver DL, Alex JC, Fairbank JT. Surgical resection and radiolocalization of the sentinel lymph node in breast cancer using a gamma probe. Surg Oncol 1993;2:335-9.

3. Wendler T, Herrmann K, Schnelzer A, Lasser T, Traub J, Kutter O, et al. First demonstration of 3-D lymphatic mapping in breast cancer using freehand SPECT. Eur J Nucl Med Mol Imaging 2010; in press.

4. Madsen EVE, Gobardhan PD, Bongers V, Albregts M, Burgmans JPJ, De Hooge P, et al. The impact on post-surgical treatment of sentinel lymph node biopsy of internal mammary lymph nodes in patients with breast cancer. Ann Surg Oncol 2007;14:1486-92.

5. Valdés Olmos RA, Vidal-Sicart S, Nieweg OE. SPECT-CT and real-time intraoperative imaging: new tools for sentinel node localizsation and radioguided surgery? Eur J Nucl Med Mol Imaging 2009;36:1-5.

6. Vermeeren L, Valdés Olmos RA, Meinhardt W, Bex A, van der Poel $\mathrm{HG}$, Vogel WV, et al. Intraoperative radioguidance with a portable gamma camera: a novel technique for laparoscopic sentinel node localisation in urological malignancies. Eur J Nucl Med Mol Imaging 2009;36:1029-36. 